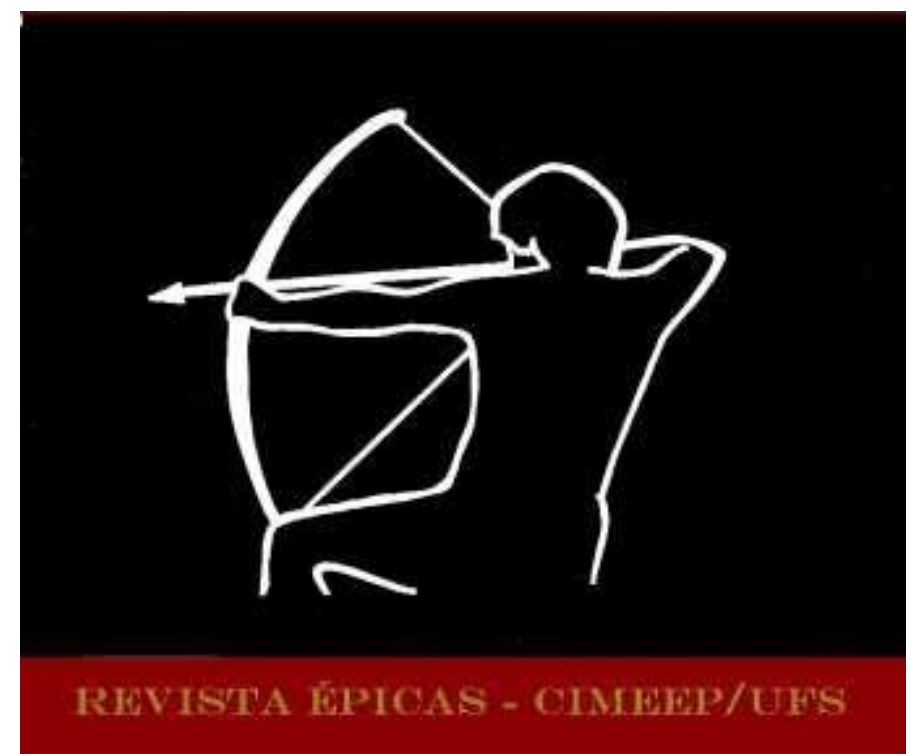

SERVAN-SCHREIBER, Catherine. De l'espace du fort a celui de la plantation. Parcours épiques bhojpuris (Inde du Nord et Île Maurice). In: Revista Épicas. Ano 5, N. 10, Dez 21, p. 16-29. ISSN 2527-080-X. DOI: http://dx.doi.org/10.47044/2527-080X.2021v10.1629

\title{
DE L'ESPACE DU FORT A CELUI DE LA PLANTATION. PARCOURS ÉPIQUES BHOJPURIS (INDE DU NORD ET ÎLE MAURICE)
}

\author{
DO ESPAÇO DO FORTE AO DA PLANTAÇÃO. \\ PERCURSOS ÉPICOS BHOJPURIS (NORTE DA ÍNDIA E ILHA MAURÍCIO)
}

Catherine Servan-Schreiber

Résumé : les épopées bhojpuries qui circulent dans le Nord de l'Inde font écho aux déplacements des hommes dans cette vaste partie du sous-continent : conquête des forts à l'Ouest par les guerriers conformément au modèle rajput, longs et périlleux parcours marchands jusqu'au Bengale et au Népal. Mais la tradition épique bhojpurie elle-même a été déplacée jusqu'à l'ile Maurice avec les coolies engagés sur les plantations, et s'est renouvelée dans ses thèmes et dans sa forme. Tout en exaltant un territoire, l'épopée ne cesse ainsi de se porter au-delà des frontières de celui-ci. Mots clés: Épopée, bhojpuri, rajput, île Maurice, coolie.

Abstract: The Bhojpuri epics that circulate in North India echo the movements of men in this vast part of the subcontinent: the conquest of forts in the West by warriors in accordance with the Rajput model, the long and perilous trade routes to Bengal and Nepal. But the Bhojpuri epic tradition itself was moved to Mauritius with the coolies hired on the plantations, and was there renewed in theme and form. While exalting a territory, the epic thus develops in the transgression of its borders.

Keywords: Epic, bhojpuri, rajput, Mauritius Islands, coolie.

«II ne faudrait pas donner une prépondérance aux personnages au détriment des espaces, importants et fouillés, que ces derniers habitent ou parcourent. II faut tenter de saisir si les lieux présents dans les films fonctionnent comme des fonds, des décors, ou bien possèdent une autonomie et un langage propres. Un langage, car ils disent souvent beaucoup, symboliquement, sur l'épisode en train de se 
dérouler", voici l'invite adressée par Amandine d'Azevedo dans son livre sur les cinémas indiens $\left(D^{\prime} A Z E V E D O, 2018\right)^{1}$. L'historienne du cinéma identifie trois espaces qui se dégagent : le village, la ville, la forêt ${ }^{2}$, puis elle se demande "si les espaces travaillés par les films proviennent d'un imaginaire mythique commun ou s'ils procèdent à des (ré)inventions (D'AZEVEDO, 2018, p. 166). Dans son analyse des rituels liés aux territoires des marges sino-indiennes, Grégoire Schlemmer fait de son côté émerger la volonté d'une emprise sur le foncier. Celle-ci s'exprime par des hiérarchisations, telles que par exemple, la préséance de premiers venus. Des mythes viennent alors renforcer les rituels (SCHLEMMER, 2012 a et b). Mais entre les mythes et les films, surgit tout le répertoire épique, propre à chaque culture régionale indienne. Les critères privilégiés pour le traitement des espaces sont-ils les mêmes ?

De manière implicite, les classifications des épopées bhojpuries de l'Inde du Nord, par les catégories qu'elles établissent - mystique, guerrière et marchande - sous-entendent que les univers épiques sont caractérisés et mis en scène par des espaces distincts. L'exemple des épopées mystiques hindoues est assez parlant, car on sait bien que le héros - un prince qui doit renoncer à son trône pour entrer dans les ordres devra suivre des itinéraires imposés, supposant un exil en forêt, la visite à de grands sanctuaires, et des pèlerinages en Himalaya, demeure bien connue de Shiva. On peut certes avoir la surprise, en regardant de plus près les textes, de découvrir, parmi les principaux espaces visités, des lieux " mixtes ", communs aux ascètes hindous et musulmans. Mais ces particularités ont été bien soulignées (BOUILLIER et CABAUD, 1989 ; GOLD, 1992 ; SERVAN-SCHREIBER, 1999), et plutôt que de revenir sur la dimension mystique, on se propose d'examiner sur trois cycles épiques différents, et de chronologie variée, la question de la représentation spatiale. Comme ces cycles sont en outre, plus que les épopées mystiques, ouverts aux destins des héroïnes, on pourra s'interroger sur les espaces parcourus dans l'épopée par des femmes.

Selon Nicole Revel, qui s'appuie sur la tradition orale palawan, l'épopée est avant tout une "quête en mariage ». Selon Florence Goyet, qui étudie des corpus épiques grecs et japonais, l'épopée a pour fonction de « résoudre une crise ». Mais comment s'articulent les notions d'espace au sein de ces définitions ?

L'espace dans les épopées n'est pas celui de la « littérature paysagiste » qui sous-tend de nombreux récits de voyage, par exemple (SALMON, 1996). Comme l'a bien montré Véronique Bouillier à propos de la perception du paysage en milieu indo-népalais, un « beau » paysage y est un paysage " utile » (BOUILLIER, 1987). On partira de plusieurs corpus épiques de l'Inde du Nord, en langue bhojpurie. Les régions de composition, de récitation et de circulation des épopées recouvrent une immense superficie. Elles se partagent sur l'Est de l'Uttar Pradesh et l'Ouest du Bihar. Au Sud, elles atteignent les frontières du Madhya Pradesh, et au Nord, elles vont jusqu'à inclure le Teraï indo-népalais. Avec deux capitales, Bénarès et Patna, le terroir bhojpuri est traversé par le Gange d'ouest en est, et cela n'est pas sans conséquence sur la tradition

\footnotetext{
${ }^{1}$ Au chapitre « Décor et surface plane : les arrière-plans et la place de la figure dans l'image ».
}

${ }^{2} C^{\prime}$ est déjà le cas pour l'univers romanesque, investi selon ces trois majeures modalités. 
orale. Anonyme, le corpus épique dans sa majorité reprend tout le fonds d'histoires, légendaires ou non, de la période médiévale. Pour les épopées guerrières et marchandes, c'est donc tout le contexte d'une société médiévale, et de sa politique de migration et de conquête de territoires qui est impliquée. Mais le bhojpuri a été aussi une langue de migration hors de l'Inde, et notamment, à l'île Maurice. Pour l'épopée bhojpurie mauricienne de plantation, cette fois, c'est le contexte d'une société coloniale du $19^{\mathrm{e}}$ siècle qui est impliqué, dans le cadre de l'engagisme, ou système d'embauche sous contrat de la main d'œuvre bhojpurie dans les îles sucrières.

Toutefois l'épopée n'est pas un genre isolé. Elle partage des structures narratives avec d'autres genres, comme la chanson populaire ou le conte de tradition orale. Pour montrer, par exemple, que de grands espaces sont parcourus, des formules toutes faites sont utilisées, ainsi dans la plainte de Udal, adressée à son frère Alha:

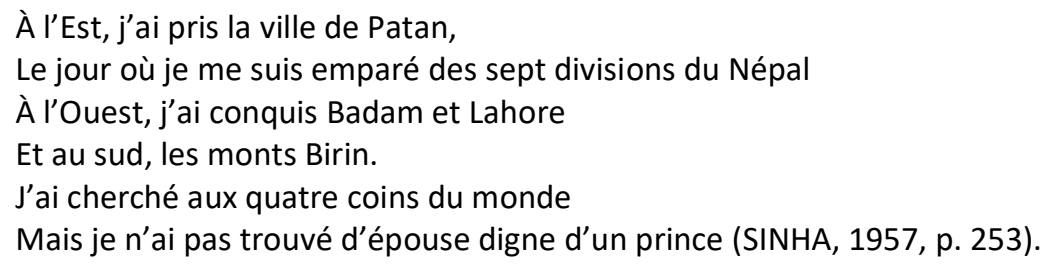

On retrouve l'usage de formules spéciales pour donner l'idée du lointain dans le conte russe Une plume de Finist le beau Sokol : « Par delà les mers lointaines, par delà les hautes montagnes, par delà trois fois neuf pays. Au trois fois dixième royaume " (ISSERLIS et AUROY, 1955). Avec la chanson populaire, l'épopée partage le goût de l'énumération des lieux parcourus. Bien souvent, des villes, mais parfois aussi, des pays, ainsi dans le célèbre konpa haïtien Lélène chérie, où le chanteur rappelle les déplacements de la diaspora haïtienne:

\footnotetext{
Nou soti New York Le'n soti New York Nou pasé Kanada Le'n soti Kanada, Nou pasé Miami Le'n soti Miami Nou pasé Paris Le'n soti Paris Nou pasé la Guyane Le'n soti la Guyane Nou pasé Gwadloup Le'n soti Gwadloup Nou pasé Porto Rico Le'n soti Porto Rico
} 
La succession des déplacements suggère que l'amour résiste à la migration et à la séparation spatiale : quel que soit le nouveau lieu investi, Lélène n'est pas oubliée, et que malgré ces périples, son retour en Haïti est attendu.

Cette technique d'énumération des villes est un procédé classique de la tradition orale bhojpurie:

\author{
Nous partirons de Chapra \\ Nous ferons halte à Ara \\ Et nous nous ferons héberger à Bagsar.
}

Ces trois villes incarnent le cœur du pays bhojpuri. Bénarès et Patna sont souvent associées, elles aussi, pour rappeler que le terroir bhojpuri englobe deux États de l'Inde, et non pas un seul, comme la plupart des autres régions. Comme dans ce chant récréatif nommé jhumar:

\author{
Je trouverai un mari à Bénarès \\ Et un sari à Patna \\ Ou encore, dans un autre jhumar, \\ Mon orfèvre est à Bénarès \\ Et mon médecin à Patna.
}

Mais l'épopée guerrière, elle, ne s'intéresse aux villes que si elles sont fortifiées. Que recouvre alors cette particularité ?

\title{
Les plaines et la symbolique des prises de forts dans la geste Rajput
}

Partant de l'idée de la " quête en épouse ", établie par Nicole Revel, on arrive de manière directe à la construction des épopées guerrières, qui relèvent de la tradition de chevalerie rajput indienne. Chaque épisode correspond à la prise d'un fort et à une alliance de mariage avec un clan rajput le plus élevé et le plus prestigieux possible. Lorsque Saraswati Joshi décrit l'épopée des Bhagrâwat et de Dev Nârâyan, elle remarque que l'espace est circonscrit à trois lieux assez proches l'un de l'autre, dans le Rajasthan : Ajmer, Mandal et Asind. Dans les épopées bhojpuries, les espaces investis sont beaucoup plus larges, et dépassent, de loin, les fiefs de la culture bhojpurie. Toutefois, les stratégies d'occupation des espaces étonnent, car elles vont dans le sens contraire des grands courants migratoires de l'Inde du Nord. En effet, le principe de migration depuis l'époque médiévale, qui part de l'Iran, du Khorassan, voire même du Yémen, pour aller jusqu'au Bengale ou en Birmanie, obéit à un schéma d'ouest en est. Voyons de plus près. Parmi les types de castes qui fournissent les héros bhojpuris désireux d'élargir leurs territoires, on trouve soit les castes martiales et pastorales des Ahirs, soit une caste de petit clan Rajput tout à fait modeste, et quasi clandestin : les Banaphar ${ }^{3}$. Les conduites de « rajputisation », c'est-à-dire d'adoption du modèle kshatriya - la classe des

\footnotetext{
${ }^{3}$ Les Banaphar, comme leur nom l'indique, viennent de la forêt (ban). Ils sont issus de l'alliance de jeunes gens rajputs avec des filles rencontrées dans la forêt et représentent donc un clan de bas statut.
} 
guerriers hindous - sont suivies par toutes ces castes modestes. Dès lors, il importe de conquérir les forts possédés par les grands clans rajputs que sont les Cauhan ou les Ujainiya. Et de s'unir aux filles de ces clans, pour renforcer le prestige d'obscures et de pauvres lignées. Or ces forts se trouvent tous à l'Ouest du territoire bhojpuri, notamment dans les villes prestigieuses de Kanauj et d'Ujjain.

Le fait de conquérir ces forts est d'autant plus important que la noblesse bhojpurie est peu reconnue, assez rebelle, et, ne disposant pas de places fortes, doit utiliser le stratagème des petites garnisons clandestines en pleine forêt. II faut alors revenir sur l'histoire du pays bhojpuri, et sur les étapes de peuplement de son terroir, étapes dont on retrouve la trace au fil des épopées.

Avant son peuplement par une population hindoue de castes, le terroir bhojpuri était en large part occupé par une population indigène tribale, vivant en forêt, les Cheros (SINGH, 1983). À partir du $12^{\mathrm{e}}$ siècle, ils sont les maîtres du Bihar et de la plaine indo-gangétique. Les Cheros sont décrits dans le Tarikh i Firuz Shâhi :

Dans cet État sauvage, on trouve des personnes sauvages elles-mêmes, hors de la civilisation. Ils habitent les forêts, et sont d'excellents chasseurs. Ils tiennent leurs assemblées sur des tapis $d^{\prime}$ herbes et de fougères dans les sous-bois. Ces gens sont habillés de plumes de paon, ils boivent dans leurs mains, ils sont nus et marchent pieds nus, ils utilisent un signal spécial si des troupes armées les attaquent, en prélevant du sang sur leur corps et en le montrant à leurs amis (SINGH, 1983 , p. 257).

Le Moyen Âge a vu cette population se faire refouler et anéantir, d'abord par les hindous de caste, puis, après la conquête musulmane, par l'alliance des chefs hindous et musulmans. Leur ennemi commun était les Cheros, et l'on s'appropriait leurs terres et leurs forêts pour augmenter et nourrir son cheptel et construire des villes fortifiées. La possession d'un fort incarnait d'abord une victoire sur les Cheros.

Par la suite, une fois les Cheros éliminés, la société guerrière bhojpurie, perçue comme assez fruste et rude, un peu héritière des tribaux auxquels elle avait arraché les terres, voulut recevoir ses lettres de noblesse à l'égal des grands clans du Rajasthan. Et c'est tout le sens des épopées guerrières comme Lorik, Alha-Udal (voir Annexe 1), ou Cuharmal. L'épopée de Lorik et celle d'Alha- Udal sont construites sur une série d'épisodes de prises de fort, lesquelles, on l'a dit, sont la condition préalable à une alliance de mariage. Suivons Lorik dans le fort de Gaura:

Écoutez l'histoire du fort de Gaura, avec ses douze hameaux Et ses 53 bazars qui s'étendent jusqu'à la ville de Kanauj Là demeurent les castes de presseurs d'huile, de vendeurs de bétel De marchands de céréales grillées et de distillateurs d'alcool D’un côté, la caste des Raghuvamsi, qui arborent leur épée à la taille De l'autre, la caste de Jaduvamsi et des Gval, qui portent leur épée cachée. Chaque maisonnée de Gaura possède sa propre salle d'armes.

Soir et matin, on s'y exerce au maniement du lejam

Il y a des terres cultivables à sept lieues alentour

Et des pâturages à perte de vue, jusqu'à quatorze lieues

C'est là que le grand héros Lorik, par la vaillance de son épée

A conquis sa renommée, depuis son camp jusque dans les trois mondes. 
Un beau fort, une belle ville, sont donc des lieux où toutes les castes sont représentées et jouent leur rôle dans la société. L'association de Lorik et du fort confirme son ascension. Simple vacher de la caste des Jaduvamsi, caste de service c'est-à-dire destinée à être au service de la noblesse, il n'a pas le droit d'arborer son épée de manière publique. Le fait d'être vainqueur d'un fort qui touche la ville de Kanauj, lui confère le prestige rajput qui lui manque. Lorik associera son nom à la prise de 4 forts, pour incarner l'idéal du guerrier rajput. De même pour Alha et Udal, du clan des Banaphar. Ils seront associés au cycle de conquête des « 52 forts ». La description du fort où réside Alha est un message lancé aux clans rajputs :

\footnotetext{
Le palais d'Alha est plein de nobles invités

Le clan des Ujjaini y tenait aussi sa cour

Neuf cents Naga, munis de clairons, y siégeaient

Il y avait aussi des clans du Marwar et du Tirhut ${ }^{4}$.

Un spectacle de danse était offert

Des danseuses de Ceylan, des danseurs de Gwalior.

Des merveilleuses danseuses du Bengale.
}

La cartographie des lieux cités dans l'épopée d'Alha Udal (Alha-Khand) a été par chance établie par Waterfield en 1875. On peut y voir Bénarès et Patna, les deux capitales bhojpuries, et le fort de Chunar, lieu stratégique car investi par la secte mystique des Kanphata Yogis, adeptes de Gorakhnath. Sa situation sur le Gange permet une mobilité différente. On voit aussi Chitor et Kanauj, deux lieux de la culture rajput en dehors du terroir bhojpuri, convoités par les héros des épopées. Mais surtout, la mention centrale reste celle du fort de Mahoba. En effet, il ne sera pas donné aux plaignants Alha et Udal de se faire adouber à Kanauj, ni de s'y faire entendre. Ils se contenteront de se rendre au fort de Mahoba, fief d'un vassal du Rajput de Kanauj, mais en s'y rendant, ils s'en empareront.

Les Banaphar sont donc "sortis de la forêt ", et cette seule métaphore reflète le manque de reconnaissance dont jouit la noblesse bhojpurie. On vante l'ardeur des recrues populaires et le grand centre d'enrôlement des armées bhojpuries à Buxar (KOLFF, 1990), plutôt que le raffinement d'une noblesse. On remarque qu'à l'exception de Chunar, les forts les plus connus du pays bhojpuris, notamment celui de Rohtas et celui de Buxar, ne figurent pas dans les épopées. Ces lieux restent associés au caractère clandestin du pouvoir bhojpuri ${ }^{5}$. Cette problématique de la transformation des cours clandestines forestières en demeures " nobles », parcourra l'épopée jusqu'à sa dernière création historique : celle de la Résistance de Kunvar Singh aux Anglais, en 1857.

\section{Un espace marchand de fleuves et de montagnes}

Loin de l'épopée rajput, l'épopée marchande met en scène les héros Bhainsaha. Ces marchands itinérants prennent la route avec leur chargement sur un bœuf, et leur geste est consignée en détail, tant

\footnotetext{
${ }^{4}$ Soit des régions situées à l'est comme à l'ouest du terroir bhojpuri, pour renforcer l'idée de l'admiration que la cour d'Alha suscite chez ses voisins.

${ }^{5}$ Jusque dans les années 1990, le fort de Rohtas, fief de la maffia bhojpurie, n'était accessible à aucune visite.
} 
dans les chants que dans les épopées. Mais les registres diffèrent, car le chant reflète une culture de l'épouse délaissée, se plaignant du départ de son époux marchand, alors que l'épopée glorifie la route prise par le Bhainsaha vers des espaces marchands à conquérir. Les échanges les plus courants se font avec le Bengale et le Népal. Comme les terres du Teraï, avec leurs marécages infestés de maladies paludéennes, sont insalubres, l'expédition est souvent jugée dangereuse. Les trajets couverts par le marchand Shobha Nayaka Banjara, dans l'épopée qui porte son nom, sont vastes. Originaire de la ville de Belupur, du district Saran, Shobha fait ses alliances vers l'est, et non l'ouest, comme les guerriers Rajput, afin de faciliter ses déplacements. Il épouse une fille du Tirhut, ou Mithila, terroir situé au Nord-Est du pays bhojpuri. Dans leurs périples, les marchands doivent subir de longs arrêts aux postes de garde (caukî). Les démêlés avec la police des douanes sont nombreux. Il leur faut affronter des pillards et autres détrousseurs de caravanes sur les routes. Aussi doivent-ils s'adjoindre les services d'un corps de soldats armés, les Burkundazé (SEN, 1977). Les trajets des caravanes sont minutieusement décrits. De Belupur, Shobha se rend à Bénarès, puis à Patna, marquant bien, dans ses premières étapes, son appartenance à la culture bhojpurie. Il s'arrête à la célèbre foire du bétail de Sonepur, sur le Gange. Et à celle de Bandih, dans le district de Ballia. Il passe au Mithila, où habitent ses beaux-parents. Il se rend à Calcutta, puis il traverse le Teraï remonte jusqu'au Morang népalais. Dans le Teraï, il suit un trajet de Gorakhpur (le grand sanctuaire shivaïte) à Bettiah, de Bettiah à Motihari, de Motihari à Sitamarhi, puis à Janakpur, et en Mithila népalais. Plus à l'est, de Patna à Hajipur, de Hajipur à Muzzafarpur, de Muzaffarpur à Darbhanga, puis à Jogbani, et à Biratnagar, où se trouvent les grandes usines de jute, et la chance d'une bonne clientèle. Ses marchandises : le riz du Bihar, des céréales, le verre, des lampes en terre, des ustensiles en terre cuite, de l'huile, des bœufs, des oiseaux en cage... De chef de caravanes, Shobha devient « roi-marchand », une figure représentative de l'ascension des commerçants Teli dans une société où l'émigration est une garantie de survie. Shobha reste parfois des années loin de son épouse à Belupur, et l'on sait que son histoire reflète une double vie marchande où l'on peut avoir une épouse en terroir bhojpurie, et une au Bengale, ou au Népal, voire en Birmanie.

Une autre projection spatiale de l'épopée concerne la relation au fleuve. Elle s'incarne dans le récit chanté de Bihula que récitent des bateliers de Bénarès et de Mirzapur. Il s'agit de montrer le lien qui unit le terroir bhojpuri au Bengale, à travers la liaison fluviale. Avec l'histoire de Bihula ${ }^{6}$ qui escorte son époux marchand décédé sur le fleuve, le barde exalte le commerce fluvial, commerce desservi par la caste des bateliers, sur des embarcations nommées les patelâ. Sont envoyés vers l'est par ce moyen des oiseaux domestiques, des animaux savants, des chèvres et du bétail plus lourd, du riz et des céréales, du sable, du ciment, mais aussi de l'opium, du salpêtre et des armes. Parallèlement aux épopées marchandes, les chansons qui évoquent ces espaces abondent. Les chants de la meule (jant), notamment, font une large place aux déplacements du commerçant bhojpuri vers le Népal, comme on peut le voir dans ce chant de la meule «Mon époux est parti pour le Morang »:

${ }^{6}$ Version abrégée et adaptée à partir de l'épopée bengalie du roi marchand Cando (Bhattacharya 1996). 
Il te faut moudre du riz ma belle, car je vais partir pour le Magadha ${ }^{7}$

Il te faut moudre du riz ma belle, car je vais partir pour le Morang.

Au Magadha, je prendrai du bétel

Au Morang, des noix de supari

Je resterai six mois au Magadha

Et je resterai bien douze mois au Morang (CHAMPION, 1983, p. 633).

\section{Epopée et genre : déplacements et migrations des héroïnes}

L'épopée bhojpuri est un univers masculin, par ses bardes, par ses compositeurs, par ses performances où seuls assistent des hommes, et par ses lieux de vente des versions imprimées, où les femmes ne s'aventurent pas ${ }^{8}$.

Pourtant, la place des trajets parcourus par les femmes non accompagnées n'est pas négligeable dans les récits. Pour comprendre les significations attribuées à ces "fictions épiques ", dans une société où voyager « akelî » (seule) pour une femme est hautement répréhensible, et rarement accordé, il faut resituer chaque héroïne dans le contexte de sa société de caste. Ainsi, une princesse rajput n'est pas supposée se déplacer seule. «Si vraiment tu me veux, viens dans mon pays, dit Rajula dans l'épopée kumaoni de Rajula Malusahi, souviens-toi, si une femme vient d'elle-même, on ne la respecte plus » (UPRETI, 2001, p. 201). Or, Canda, de noblesse rajput, brave les conventions dans l'épopée bhojpurie de Lorik. Traversant une dangereuse forêt, elle retourne seule au village de ses parents, transgressant deux fois les règles qui s'appliquent au statut de la femme mariée ${ }^{9}$. En attribuant à Canda les qualités masculines d'autonomie et de hardiesse, en la plaçant en pleine forêt, l'épopée fait l'héroïne une femme intrépide, donc parée de séduction, à l'inverse de Manjari, l'épouse de Lorik, qui reste dans le périmètre de sa demeure et de ses champs, élève ses enfants, surveille le bétail, s'occupe de sa belle-famille, et perdra son mari par son caractère trop « domestique ». De la même façon, Sundari, en tant qu'épouse du marchand Shobha Nayaka Banjara, n'est pas supposée partir sur ses traces et doit se contenter de l'attendre à la maison, quel que soit le temps d'absence à subir. Aussi Sundari use-t-elle d'un stratagème bien connu : s'habiller en homme, pour suivre l'itinéraire emprunté par son époux, et tenter de le rejoindre. Dans l'épopée de Bihula, Bihulâ se lance hardiment sur le fleuve pour un long périple en compagnie du corps défunt de son mari.

Ces femmes, qui dérogent à la tradition, en franchissant des espaces lointains, seules, font l'objet d'admiration dans l'auditoire, mais le barde rappelle aussi toutes les mésaventures sexuelles qu'elles peuvent subir, notamment de la part de bateliers dont la réputation est assez noircie par la tradition orale. En revanche, dans le cas de Hirni et Birni, deux sœurs de basse caste, issues des Nat, aucun interdit de déplacement ne pèse sur elles, et c'est sans embûche ni encombre qu'elles parcourent d'immenses distances

\footnotetext{
${ }^{7}$ Le Magadha est la région au sud de Videha, sur la rive droite du Gange, terroir voisin. « Morang » désigne district du Teraï népalais entre les rivières Kosi et Meci, réputé pour être infesté par la malaria.

${ }^{8}$ Du fait que les épopées voisinent sur l'étalage avec de petits manuels érotiques.

${ }^{9}$ La femme ne doit pas se déplacer seule, et elle ne doit pas retourner habiter chez ses parents après son mariage
} 
en compagnie de leur bœuf et d'un coq de combat. Partant du Gujarat, elles arrivent en terroir bhojpuri, dans un trajet qui reflète bien la migration des populations d'ouest en est. Ces trajets leur assureront la respectabilité, puisqu'elles finiront par s'établir et faire alliance avec un membre d'une caste supérieure à elle. La migration est là encore, condition de gagne-pain et de survie. Toutefois, aucune femme ne sera peinte dans la « traversée des Eaux noires » et la sortie du terroir bhojpuri par la mer, que narre l'épopée des coolies.

\section{L'espace de la plantation comme " une vallée de larmes " : une histoire de coolies}

Au moment de quitter l'Inde pour les îles sucrières, notamment Maurice, les engagés emportent avec eux la tradition des gâthâ (épopées). Sous forme de mémoire orale, ou sous forme de petit livret de colportage. Alha-Udal, Gopicand, Lorik sont attestés à l'île Maurice. Lorik est la plus récitée, car la population Ahir (dite aussi Yadava) est largement représentée, et ses loisirs sont bien décrits (BOODHOO, 1999). Mais le bhojpuri, tout en étant largement parlé à Maurice, ne jouit pas d'un statut littéraire très affirmé. On l'utilise surtout dans le cadre de la chanson populaire. En matière d'épopée, de nouvelles créations voient le jour, comme celle composée par le « poète national » (rashtriya kavi) Bhagat Madhukar (voir Annexe 2). Bien que composant de nombreux chants en bhojpuri, il préfère rédiger son épopée en hindi.

Cette épopée a été composée en 1968, un an exactement après l'indépendance de Maurice, pour rappeler le passé de l'engagisme à une époque où la communauté indienne bhojpuri avait du mal à assumer ce contexte de la migration. Il s'agit d’une "mini épopée » versifiée (" $A$ mini epic on the coolies in Mauritius ", voir Annexe 3), rédigée en douze parties. Comme pour reprendre le flambeau de la tradition épique bhojpurie là où s'est arrêtée sa dernière composition (l'épopée de Kunvar Singh), Madhukar place I'action en 1857, au moment de la Mutinerie, et met en scène les hommes bhojpuri qui ont bravé l'autorité du colonisateur et qui, dans la nécessité de fuir, ont embarqué pour l'ailleurs en se rendant à Calcutta. Ils recevront le nom de " girmitiya $»^{10}$ ou de Kalkatiya ${ }^{11}$, ou " angagé ${ }^{12}$, ou coolie. Madhukar identifie trois principaux espaces de vie du migrant bhojpuri. Tout d'abord, la traversée des Océans. Curieusement, il ne fait pas allusion aux Eaux Noires et n'utilise pas l'expression de "Kala Pani ». II parle de samudr yatra, le voyage en mer interdit, au-delà des terres. C'est l'odyssée du " malheureux migrant », " pardes becara ». Les conditions de transport sont rudes, et les éléments se déchainent. Le voyage sur l'Océan relève du sacrifice (balidan), et non du blâme pour avoir enfreint un espace interdit ${ }^{13}$. Les larmes du coolie abondent dès ce voyage. Le second espace mis en scène est celui de Port-Louis où le navire accoste. Et là, entre en jeu la fiction de la découverte du monde blanc et du régime colonial. Alors même que l'Inde des années 1834 à 1880, années de l'engagisme, vivait sous un régime colonial britannique, la légende des coolies veut qu'ils

\footnotetext{
${ }^{10}$ Ce terme vient du mot anglais « agreement », par allusion au contrat qui les engage.

11 «Qui vient de Calcutta».

12 L'engagisme est le système d'embauche de cinq ans sous contrat.

13 Edouard Glissant et Patrick Chamoiseau ont rappelé que l'Océan Indien et la mer des Caraïbes sont des cimetières (2009).
} 
découvrent avec stupeur et effroi le monde colonial en arrivant à Maurice ${ }^{14}$. Toutefois, les colons n'y sont pas seulement anglais, mais français.

Dans la ville de Port-Louis, on pouvait voir les maisons et les Bureaux des Blancs. Des Malgaches, des Zoulous, des esclaves africains y travaillaient chaque jour comme esclaves Et le patron français régnait sur eux (GOYANKA, 2003, p. 143).

Puis vient l'espace lui-même de la plantation, et de ses baraquements (kothî). On attribue les coolies à chaque plantation : untel chez Monsieur Manes, untel chez Monsieur Hardy, untel chez Monsieur John, untel à la plantation Beau-Rivage. La haine du patron français et du contremaître rejaillit dans beaucoup de chansons populaires bhojpuries comme "L'usine de monsieur Manes a pris feu ». La crainte du colon nourrit l'épopée. Des manques se font sentir sur la plantation : pas d'école, pas de temple. On y est mal nourri et on attrape des maladies comme la malaria. Madhukar veut faire de sa « mini épopée » un nouveau chant : naval git, pour montrer le chemin parcouru et honorer le nom des coolies qui se sont sacrifiés en quittant les rivages de l'Inde.

Nourri du Ramayana de Tulsi Das, Bhagat Madhukar connait aussi toute la dimension épique (vîr gâthâ) de la tradition orale bhojpuri. Il écrit aussi nombre de poèmes sur l'engagisme, comme " Les larmes du coolie, Kulî ki ânsû, en 1970. Dans ces poèmes apparaît un espace important, celui du jardin : le jardin offre une compensation au monde de la plantation, où le coolie a un endroit intime à lui, et peut cultiver des fleurs $^{15}$. Ainsi le poème Phûlbagiyâ, « Le jardin de fleurs »:

Ohi phulbagiyâ mein kya kya phule?

Campâ, cameli, gulnar, jiya cahat dekhan ko » (GOYANKA, 2003, p. 170)

Qu'est ce qui fleurit dans ce jardin?

Des fleurs de magnolia, des jasmins, des roses, que l'esprit aime à voir.

Ainsi deux espaces s'opposent, celui, épique, de la plantation où règne la souffrance, et celui du jardin, rappelant l'Inde, où règne la beauté et l'harmonie.

\section{Conclusion}

Différentes logiques spatiales parcourent l'univers épique bhojpuri. Mais elles évoluent aussi en fonction des chronologies. À la période médiévale, la confrontation des sociétés de caste, surtout les castes martiales rajputs et les castes pastorales Ahirs avec les populations tribales - confrontation qui dura 300 ans - voit l'obsession de conquêtes de forts dessiner une nouvelle géographie pour empiéter sur la forêt et implanter une agriculture à la place de la chasse et de la cueillette. Mais, l'épopée nous le rappelle, rien n'est acquis facilement, car les terres gardent la mémoire du passé conflictuel:

\footnotetext{
${ }^{14}$ Alors qu'ils sont issus de sociétés où l'exploitation coloniale du jute, de la canne à sucre ou de l'indigo est déjà en place, et qu'ils connaissent parfaitement.

${ }^{15}$ Sur le thème du jardin tropical investi par l'engagé indien dans les îles sucrières, voir la thèse en cours de Lou Kermarrec.
} 
Sur les terres cultivables, toutes sortes de mauvais esprits rodent Fantômes et revenants.

Sur les pâturages, rodent mille six cents trépassés malfaisants, Les plus braves guerriers en ont les poils hérissés de peur (Lorik).

À partir de la fin du $18^{\mathrm{e}}$ siècle, la montée progressive des castes de Teli et Baniya installe un double système de résidence et de vie de famille pour ces marchands. 1834 crée une nouvelle étape pour les déplacements de main d'oeuvre, des liens nouveaux se font avec d'autres pays que l'Inde, notamment, les îles sucrières.

L'avantage de pouvoir utiliser les sources offertes par les corpus épiques, est de documenter des pans manquants de l'histoire. Ainsi les travaux de Claudine Salmon (1996) ou ceux de Claudine Le Blanc et Jacques Weber (2009), montrent bien que la plupart des récits de voyage hors de l'Inde viennent d'auteurs musulmans. Le corpus épique renforce les recherches menées sur l'engagisme en introduisant de nouveaux auteurs du milieu hindou. Les populations croisées le sont dans un contexte d'antagonisme. Conflits pour la terre avec les Cheros, puis avec les musulmans. Haine du colon français. Mais les alliances peuvent changer, ainsi, dans l'épopée d'Alha Udal, Alha Udal et le leader musulman de Bénarès, qui étaient en procès, font alliance contre un autre roi. Sans aller jusqu'à intégrer l'opposition " guerrier/marchand " suggérée par Harald Tambs-Lyche dans son analyse de l'épopée (TAMBS LYCHE, 1996), on relève que les communautés mentionnées par l'épopée investissent chacune leur espace d'une manière différente. À l'ouest, on gagne en prestige, à l'est, on s'enrichit. L'Inde est présentée par ses historiens comme une terre "d'absolue mobilité » (MARKOVITS, POUCHEPADASS et SUBRAHMANYAM, 2003). Et à cet égard, on aurait une vision trop partielle des mobilités bhojpuries si on ne consultait que l'un des trois registres épiques. On voit que par rapport à l'analyse des films indiens, les espaces ville/village/forêt ne sont pas les références mises en place. Le fleuve, la montagne, le lointain, la défense du territoire fortifié occupent les descriptions. De ce fait, l'épopée porte en elle des contradictions, car elle vise à la fois à créer le sentiment d'un terroir partagé, et à valoriser les déplacements hors de cet espace, en donnant du souffle et du panache à la migration. Aussi doit-on prendre en compte la notion de frontière linguistique et culturelle, comme le prescrit Daniel Nordman dans son approche des frontières de France. C'est ce qui se passe aux frontières du terroir qui définit ce terroir. Le fait que la performance de l'épopée déborde le terroir pour se produire à ses marges renforce cette visibilité d'un espace commun. Au final, l'espace qui crée et rappelle l'identité bhojpurie est celui où l'épopée se dit.

\section{Références bibliographiques}

BHATTACHARYA, France. Le voyage dans la littérature médiévale du Bengale. Itinéraires obligés et modèles idéologiques. In: SALMON, Claudine (ed). Récits de voyage des Asiatiques. Genres, mentalités et conceptions de l'espace. Paris: EFEO, 1996, p. 39-52.

BOODHOO, Sarita. Bhojpuri Traditions in Mauritius. Port-Louis: Mauritius Bhojpuri Institute, 1999. 
BOUILLIER, Véronique; CABAUD, Marie-Christine. Les aventures du Prince Dikpal. Cahiers de l'Asie du SudEst, 25, 1989, p. 19-42.

BOUILLIER, Véronique Râmro-narâmro. La perception du paysage chez les Indo-Népalais des collines du Népal central. Études rurales, $n^{\circ} 107-108,1987$, p. 43-53.

CARTER, Marina. Across the Kalapani. The Bihari presence in Mauritius. London: Centre for Research on Indian Ocean Societies, 2000.

CHAMPION, C. Chants villageois du pays bhojpuri. Littérature orale de I'Inde du Nord. Université Paris III, thèse de $3^{\mathrm{e}}$ cycle en études indiennes, 1983.

CHAMPION, C. Un aller et retour au paradis de I'Inde. Le voyage au Bihar dans l'hagiographie islamique. In : SALMON, Claudine (ed.). Récits de voyage des Asiatiques. Genres, mentalités et conceptions de l'espace. Paris: EFEO, 1996, p. 53-67.

CHAMOISEAU, Patrick; GLISSANT, Edouard. L'Intraitable beauté du monde. Editions Galaade, Institut du Tout-Monde, 2009.

GOLD, Ann Grodzins. A Carnival of Parting. The Tales of King Bhartrihari and King Gopicand as Sung and Told by Madhu Natisar Nath of Ghatiyali, Rajasthan. Berkeley: University of California Press, 1992.

GOYANKAR, Kamal Kishor. Brajendra Kumar Bhagat 'Madhukar' kâvya rachnâvalî. Delhi: Natraj Prakashan, 2003.

GOYET, Florence. Penser sans concepts. Fonction de l'épopée guerrière. Paris : Honoré Champion, 2006.

ISSERLIS, H. ; Auroy, J. Contes de Russie. Paris : Hatier-Boivin, 1955.

JOSHI, Saraswati. L'épopée des Bagrâwat et de Dev Narayan. In : REVEL, N. ; CHAMPION, C. (eds.). Les Littératures de la Voix. Épopées et récits de fondation. INALCO, CRO, 1994-95, p. 23-30.

KAUSHAL, Molly (ed.). Chanted Narratives. The Living Katha Vachana Tradition. New Delhi: Indira Gandhi National Centre for the Arts, 2001.

KOLFF, Dirk. Naukar Rajput and Sepoys. The Etnohistory of the Military Labour Market in Hindustan. Cambridge: Cambridge University Press, 1990.

LE BLANC, Claudine WEBER, Jacques (eds.). L'ailleurs de l'autre. Récits de voyageurs extra-européens. Rennes, Presses universitaires de Rennes, 2009.

MARKOVITS, Claude; POUCHEPADASS, Jacques; SUBRAHMANYAM, Sanjay (eds.). Society and Circulation. Mobile people and Itinerant Cultures in South Asia, 1750-1950. Delhi, Permanent Black, 2003.

NORDMAN, Daniel. Frontières de France. De l'espace au territoire, $\mathbf{X V I}^{\mathrm{e}}-\mathbf{X I X} \mathbf{X}^{\mathrm{e}}$ siècles. Paris: Gallimard, Bibliothèque des Histoires, 1999.

REVEL, Nicole. La quête en épouse, une épopée palawan chantée par Mäsinu, The Quest for a Wife. A palawan Epic sung by Mäsinu, Mamiminbin. Paris : UNESCO/Langues et Mondes, L'Asiathèque, 2000.

SALMON, Claudine (ed.). Récits de voyage des Asiatiques. Genres, mentalités et conceptions de l'espace. Paris : EFEO, 1996.

SCHLEMMER, Grégoire. Rituels, territoires et pouvoirs dans les marges sino indiennes. Moussons, 19, 2012a, p. 5-18.

SCHLEMMER, Grégoire. Fils du territoire, alliés de la forêt. Expression rituelle du rapport au territoire chez les Kulungs Rais du Népal Oriental. Moussons, 19, 2012, b, p. 33-50.

SERVAN-SCHREIBER, Catherine. Partage des sites et partage des textes. Un modèle d'acculturation de l'islam en Inde. In: ASSAYAG, J.; TARABOUT, G. (eds.). Altérité et identité. Islam et christianisme en Inde, Purusartha, $n^{\circ} 19,1997$, Paris, éditions de l'EHESS.

SERVAN-SCHREIBER, C. Chanteurs itinérants. La tradition orale bhojpuri. Paris : L'Harmattan, 1999. 
SINGH, K.S. Medieval tribal Bihar. In: ASKARI, S.H.; AHMAD, Q. (eds.). The Comprehensive History of Bihar. vol. II, part 1, Patna: Kashi Prasad Jayaswal Research Institute, 1983, p. 255-289.

SINHA, Satyavrata. Bhojpuri Lok gatha. Allahabad: Hindustani Akademi, 1957.

TAMBS-LYCHE, Harald. Une tradition orale face au post-modernisme: I'exemple des bardes du Saurashtra", in C. CHAMPION, C. (ed). Traditions orales dans le monde indien, Purusartha. Paris : Editions de l'EHESS, 1996, p. 23-37.

TAMBS-LYCHE, Harald. Réflexion sur les mythes de fondation des royaumes rajput. In : REVEL, N. ; CHAMPION. C. (eds.). Les Littératures de la Voix. Épopées et récits de fondation. INALCO: CRO, 1994-95, p. 37-54.

TEELOCK, Vijaya. Angagé. Explorations into the History, Society and Culture of Indentured Immigrants and their descendants in Mauritius. Port-Louis: Aapravasi Ghat Trust Fund, 3 volumes, 2012.

UPADHYAYA, K.D. Bhojpuri Lok Git. Prayag: Hindi Sahitya Sammelan, 2 vols, 1956-57.

UPRETI, Mohan. Rajula-Malushahi, the Oral Epic (ballad) of Kumaon. In: KAUSHAL, Molly (ed.). Chanted Narratives. The Living Katha Vachana Tradition. New Delhi: Indira Gandhi National Centre for the Arts, 2001, p. 197-203.

WATERFIELD, William; GRIERSON, George. The Lay of Alha. A Song of Rajput Chivalry as sung by the Minstrels of Northern India. Gurgaon: Vintage Books, 1990.

\section{Annexe 1:}

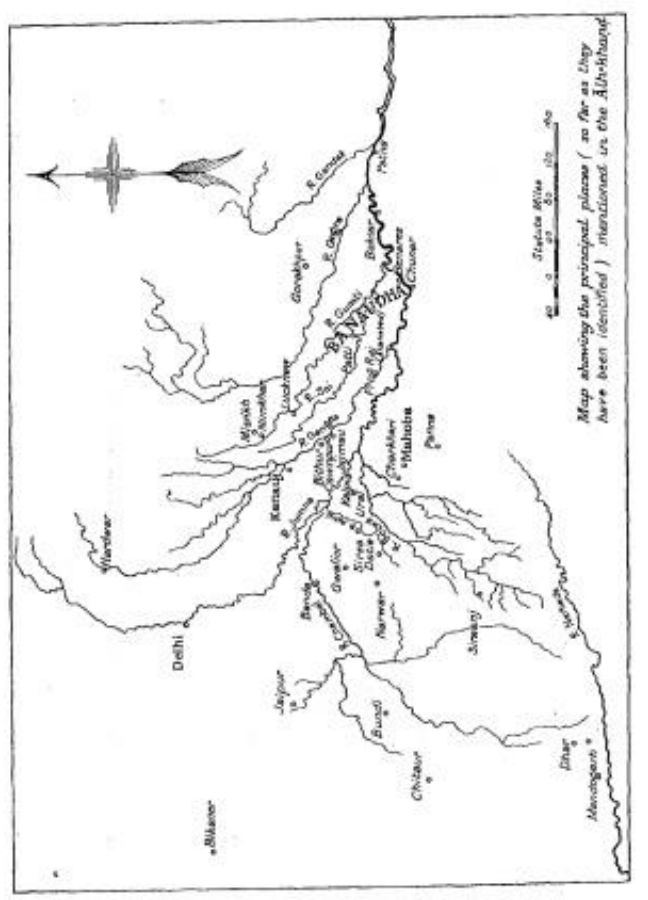

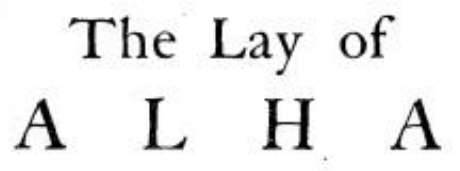

A Saga of Rajput Chivalry as sung by Minstrels of

Northern India

Partly translated in English Ballad Metre by the late WILLIAM WATERFIELD of the Bengal Croil Service

With an Introduction and Abstracts of the untranslated Portions

by Str GEORGE GRIERSON, K.C.I.E. 
Annexe 2:

कुआँ भी एक अदुमुत था जिसका नीर निर्मल था, वहीं एक शान्त कुटिया में नरायण भक्त रहता धा । सकल परिवार मिलकर के सुख्द्ध जीवन बित्ताते थे सदा ही बैन की बंशी बिना संशय बजाते थे ।

तीसरा अध्याय

(सन 1857 की क्रान्ति)

परन्तु एक दिन भाई छिद्धा संग्राम भारत में

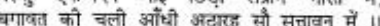
जगी बम्बई, जगी दिल्ली, जगा मेरठ, जगी झाँसी, लगा स्वाधीनता नारा करोड़ों जन चहे फॉसी । लड़े कितने बढे कितने अड़े कितने गड़े कितने धिरे कितने गिरे कितने जले कितने मरे कितने जली कुटिया नरायण की, जली खेती किसानों की जली रोटी मजूरों की, जली किस्मत गरीबों की चला तकदीर को लेकर धरम की गाँड को लेकर, परम प्रिय पुत्र कधे पर करम का वीर कलकक्ता ।

\section{चौथा अध्याय}

(अरकावी का जाल)

कहीं पर ट्रम चलता था कहीं फिटनों की पँची थीं कहाँ रंगीन बल्वों से सीी थी डीय को माता । कहीं गडबड झमेला था कहीं सैदान में मेला था मगर इस जन - समुद्दर में खड़ा कुली अकेला था

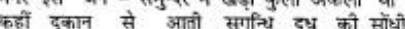
कहीं हूलबा, रसोगुल्ला परतां से बुलाते थे

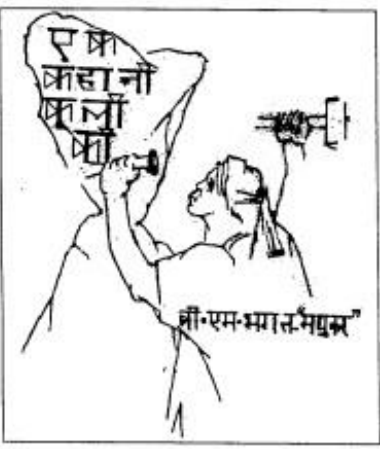

'एक कहानी कुली की' के प्रथम संस्करण का आवरण $142 /$ ब्रजेन्द्र कुमार भगत 'मघुकर' काव्य-रचनावली
थी आय आँवों में नयन से आँकती ज्ञाता, परन्तु बाप - बेटे का चणा ही बस घबेना था अनेकों दिवस ऐसे ही मजूरी दूँढ़ते बीते वरन्तु भाग्य के खोटे बिताये से मू लोटे भला होनी कमी भी चुप लगाकर बेच सकती है ? कहाँ से एक बाब् बन सुनहरा जाल रचती है गवारों को गुरीबां को मुसीबत गुम के मारों को सहल्लों बेसहारों को लुभाती है फँसाती है सुनो अर्जा मेर भाई़ ! चलो मौरच मेंरे भार्ई वहाँ बिलकुल न रोना है वहाँ सोना ही सोना है बहाँ आराम फरना है नहीं कुछ काम करना है वहाँ चाँदी बरसती है वहाँ किस्मत चसकती है वहाँ संगीत सरगम है वहाँ पनरयाम मधन्य है

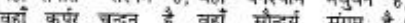

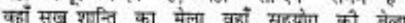

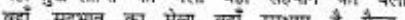

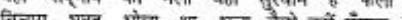
धिचारा मक्त मोता था मला केसे नहां फसता ? करम की कुटिल चालो में भयंकर मृत्यु आलों में गया वह एक दूरी में की लिरमेट बन्चन में लिया कुछ नोट गठरी में, दिवा गृह-त्पाग पल-दिन में ।

पाँचवाँ अध्याय

(समुद्र-यात्रा)

दिबस का अन्त था आया गगन में लालिमा छाई दिबाकर तरस खाकर के छुपा सागर की गोदी में । नदी हुगली की जोटी पर लग्गा सागयान की गोदी में की

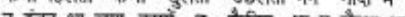
न इसन था लगा उसमे 7 कीबिन था 7 सैलून था, तने थे पाल रस्सी से पवन का आसरा बस्त धा हदय में पीर लेकर के नयन में दारकर आाँस चढ़े कूली सवा दो सौ, चले परदेश बेचारे न था बाबा, न थी बेटी, न था बेटा, न मरतारी, न था बन्धु कुटुम कोई रही बस साथ लाचारी सभी को छ्वेक पर रहना पडा पशुओं की पाँती में, सभी को साध में सोना पड़ा तिब्यत की छावी में सड़े चावल, सड़ी मछली, सडी दाबे, कड़ी गोडी, धधकती शे के आगे उतापी काज के योी

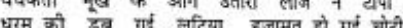
धरम की ने बहलो के माईई जान कपनी सिन्दु मानस में किती में घटा काली भवानक रात तूफानी,

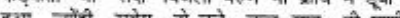
हुआ ज्योड़ी सकेग तो बचे कुल साठ ही प्राणी, मिले बाकी रसातल में नहां किर लीट कर आये बचाओ रे ! बचाओ रै ! जहाजी एक चिल्लाया महा 'शारक' निगलने को भयंकर यंत दिखलाये लगे सब काँपने डर से ठिले अपने नहीं दर से धड़ाधड़ भेड़-यकरी का किया बलिदान नाविक ने।

Annexe 3:

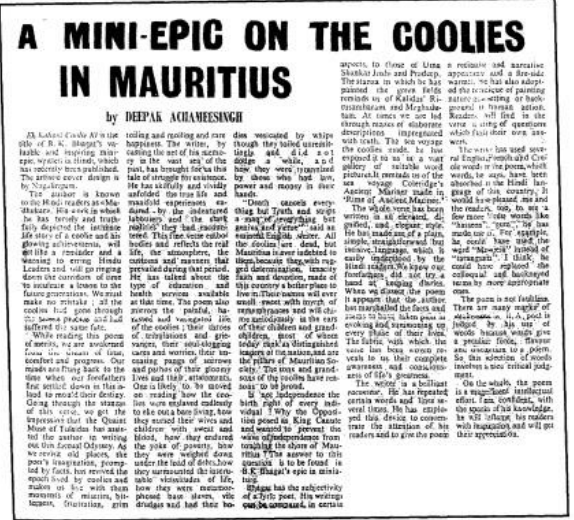

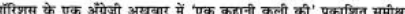

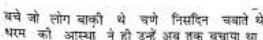

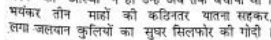

छठा अध्याय
(कोठी में पद्षण्या)

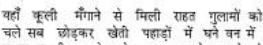

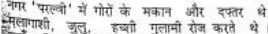

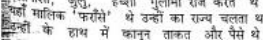

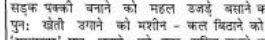

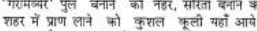

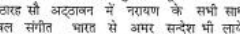

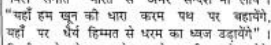

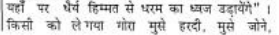

एक कहानी कुती की / 143 